\title{
Epidemiology and genetic diversity of human parechoviruses circulating among children hospitalised with acute gastroenteritis in Pune, Western India: a 5-years study
}

\author{
P. R. PATIL, N. N. GANORKAR AND V. GOPALKRISHNA* \\ Enteric Viruses Group, National Institute of Virology, 20-A, Dr Ambedkar Road, Pune 411001, India
}

Received 2 April 2017; Final revision 30 September 2017; Accepted 1 November 2017; first published online 27 November 2017

\section{SUMMARY}

Human parechoviruses (HPeVs) are known to cause various clinical manifestations including acute gastroenteritis. Although $\mathrm{HPeV}$ infections and their genotypes have been detected in human patients worldwide, no such reports are available from India to ascertain the association of HPeVs in acute gastroenteritis. The present study was conducted to determine the clinical features and genetic diversity of HPeVs detected in children hospitalised for acute gastroenteritis. Stool specimens $(n=979)$ collected from children aged $\leqslant 5$ years hospitalised for acute gastroenteritis in Pune, western India during January 2006-December 2010 were included. HPeV RNA was detected by reverse transcription-polymerase chain reaction (RT-PCR) (5'UTR) followed by genotyping using VP1 gene-based PCR and phylogenetic analysis. HPeV was detected in $13 \cdot 9 \%(136 / 979)$ of the cases, co-infections with other enteric viruses were found in $43 \cdot 4 \%$. HPeV was more frequent in children $\leqslant 1$ year age with infections reported throughout the year. A total of 102/136 (75\%) HPeV strains were genotyped, which comprised 13 different $\mathrm{HPeV}$ genotypes. Of these, HPeV1 was the most predominant genotype detected and phylogenetically clustered with the Harris strain which is rarely reported. The study documents circulation of heterogeneous $\mathrm{HPeV}$ genotypes. Two variant strains of HPeV4 and 'RGD absent' HPeV5 and 6 strains were also detected. This is the first report of $\mathrm{HPeV}$ with diversified genotypes identified in acute gastroenteritis patients from India.

Key words: Acute gastroenteritis, clinical features, genotypes, human parechoviruses, prevalence.

\section{INTRODUCTION}

Human parechoviruses (HPeVs) of the family picornaviridae are small, non-enveloped, positive sense, singlestranded (ss) RNA viruses. These viruses, previously known as Echo 22 and 23, were first isolated from the children during a summer outbreak of diarrhoea [1]. Subsequently, genomic analysis revealed them to be distinct from Echoviruses and classified into a new genus

\footnotetext{
* Author for correspondence: V. Gopalkrishna, Enteric Viruses Group, National Institute of Virology, 20-A, Dr Ambedkar Road, Pune 411 001, India.

(Email: gopalvk58@hotmail.com)
}

parechovirus [2]. The HPeV genome is $\sim 7 \cdot 4 \mathrm{~kb}$ nucleotides in length and contains a large open reading frame coding for a single polyprotein which is post-translationally cleaved into three structural (VP0, VP3 and VP1) and seven non-structural (2A-2C and 3A-3D) proteins [3]. Based on the VP1 gene analysis, $\mathrm{HPeVs}$ have been classified into 17 genotypes i.e., HPeV1-17 (http://www.picornastudygroup.com/types/ parechovirus/hpev.htm).

$\mathrm{HPeVs}$ are widely distributed and have been reported to cause various clinical manifestations including gastroenteritis, upper respiratory tract infections, sepsis-like illness, aseptic meningitis, Reye's syndrome, myocarditis, encephalitis and transient 
paralysis [4]. Among HPeVs, $\mathrm{HPeV} 1$ is a widespread pathogen most commonly associated with gastrointestinal and respiratory symptoms and less frequently reported in severe disease conditions, encephalitis and flaccid paralysis [5]. HPeV2 infections rarely occur and are associated with gastrointestinal symptoms [6]. HPeV3 and HPeV4 were isolated from neonates with sepsis and also with high-grade fever and poor feeding, respectively [5, 7]. HPeV5 formerly classified as HPeV2 by neutralisation test has been reclassified on the basis of phylogenetic analysis of the VP1 region [8]. HPeV6 was isolated from a child suffering from Reye's syndrome [9] while $\mathrm{HPeV} 7$ has been detected in a healthy child having close contact with a person who had acute flaccid paralysis [9, 10]. $\mathrm{HPeV} 8$ was isolated from a child with enteritis during an outbreak of diarrhoea in Brazil [11]. HPeV9-13 were discovered in Thailand while HPeV14 was in the Netherlands. HPeV 15 and 16 have been reported recently from Bolivia [12].

Acute gastroenteritis (AGE) is a global health problem and a major contributor to childhood morbidity and mortality worldwide. Approximately two million deaths occur due to acute gastroenteritis in children under 5 years of age in the developed and developing countries [13]. In India, an association of enteric viruses such as rotavirus (RV), norovirus $(\mathrm{NoV})$, adenovirus (AdV), astrovirus (AstV) and other picornaviruses with acute gastroenteritis has been documented [14-18]. Although variable rates (1.6-29.4\%) of $\mathrm{HPeV}$ infections and their genotypic distribution in acute gastroenteritis cases have been reported worldwide [19], no such reports are available from India. The present study was aimed to gain insight into the epidemiological features and genetic diversity of $\mathrm{HPeV}$ strains circulating in hospitalised children with acute gastroenteritis. This is the first report that describes the epidemiological and clinical features and genetic characterisation of $\mathrm{HPeVs}$ in acute gastroenteritis cases from India.

\section{METHODS}

\section{Specimens}

Stool specimens $(n=979)$ collected from children, aged $<1-60$ months (median age 12 months), hospitalised for acute gastroenteritis in Pune, western India during January 2006-December 2010 were included in the study. A sporadic case of acute gastroenteritis was defined as $\geqslant 3$ episodes of diarrhoea reported within
$24 \mathrm{~h}$ with or without associated symptoms such as vomiting, fever and abdominal pain. One specimen per patient was collected for this study within $24 \mathrm{~h}$ of hospitalisation and prior written informed consent was taken from the parents/guardians. All the patients were examined for fever, number of episodes and duration of vomiting and diarrhoea, the extent of dehydration and treatment for the assessment of disease severity [20].

\section{Nucleic acid extraction and detection of $\mathrm{HPeV}$ and other enteric viruses}

Viral nucleic acid was extracted from 30\% (weight/ volume or volume/volume) of the stool suspended in $0.01 \mathrm{M}$ phosphate buffered saline (PBS) $\mathrm{pH} 7.2$ by using QIAamp viral RNA mini kit (Qiagen, Hilden, Germany) and stored at $-80{ }^{\circ} \mathrm{C}$ until used. Presence of HPeV-RNA was identified by reverse transcriptionpolymerase chain reaction (RT-PCR) using $5^{\prime} \mathrm{UTR}$ specific primers followed by genotyping with primers specific for VP1 gene [21, 22].

The specimens were also tested for the presence of group A rotavirus antigen by ELISA (Dako Cytomation, Ely, Cambs, UK) and NoV, AdV, AstV and EV nucleic acids by RT-PCR/PCR as described earlier [15-17, 23].

All the PCR products were electrophoresed in 2\% agarose gel containing ethidium bromide $(0.5 \mu \mathrm{g} / \mathrm{ml})$, visualised under UV transilluminator and products were sequenced with an ABI Prism 3700 DNA Analyser (Applied Biosystems, USA).

\section{Nucleotide sequence analysis of $\mathrm{HPeV}$ genes}

Sequence identity of VP1 gene of the HPeV strains was determined through BLAST analysis (http://www.ncbi. nlm.nih.gov/blast). Multiple sequence alignment was carried out using CLUSTAL W and phylogenetic analysis was carried out using MEGA 5.0 and the tree was generated with maximum likelihood method and Kimura 2-parameter distance model. The reliability of the phylogenetic tree was tested by applying the bootstrap test with 1000 bootstrap replications [24]. The nucleotide sequences reported in this study have been deposited in GenBank under accession numbers KJ743617-KJ743718.

\section{Statistical methods}

The descriptive statistical analysis was done in Microsoft Excel 2007 while the prevalence between 
different groups was compared using $\chi^{2}$ tests. All these tests were performed at the significance level of 0.05 using Open Epi 2.3.1 version and all those tests were two-tailed. Odds ratios (OR) and 95\% confidence intervals (CIs) were also calculated.

\section{RESULTS}

\section{Prevalence of $\mathrm{HPeV}$ among children with acute gastroenteritis}

At least one enteric virus was detected in $602(61 \cdot 5 \%)$ of the 979 stool specimens collected from the children hospitalised for acute gastroenteritis in Pune, western India during 2006-2010.

Overall, RV, EV, NoV, AdV and AstV were detected in $242(24 \cdot 7 \%), 118(12 \cdot 1 \%) 65(6 \cdot 6 \%), 29(2 \cdot 9 \%)$ and 12 $(1 \cdot 2 \%)$ of the specimens respectively. HPeV was detected in $13.9 \%(136 / 979)$ of the specimens tested which comprises $56 \cdot 6 \%$ (77/136) of HPeV mono infections and $43 \cdot 4 \%$ (59/136) co-infection of $\mathrm{HPeV}$ with other enteric viruses. Among co-infections, dual infections were detected in $52(88.13 \%)$ and triple infections in seven $(11 \cdot 86 \%)$ of the specimens. Rota virus (33.9\%) and enterovirus $(32 \cdot 2 \%)$ were detected most frequently along with $\mathrm{HPeV}$.

The overall prevalence of lone HPeV among acute gastroenteritis cases which are negative for other enteric viruses was observed to be $14 \cdot 1 \%$ (77/548) (Table 1). The yearly prevalence of $\mathrm{HPeV}$ in stool specimens varied during the study period. $\mathrm{HPeV}$ was detected in $9.6 \%$ and $10.7 \%$ of the patients during 2006 and 2010, respectively and $6-8 \%$ in the remaining years (Fig. 1).

\section{Epidemiological characteristics of $\mathrm{HPeV}$ infection among children with acute gastroenteritis}

Age of the HPeV positive children $(n=136)$ was in the range 1-54 months (median, 10 months). The detection rate of $\mathrm{HPeV}$ decreased with the increase in their age and it was found more frequent in children $\leqslant 1$ year of age. Among children in $\leqslant 1$ year of age group, HPeV positivity was found significantly high $(68.8 \%)$ as compared with the children tested positive for other enteric viral infections $\left(\chi^{2}=4.3 ; P<0.05\right.$; $\mathrm{OR}=1 \cdot 8)$ and negative for all enteric viral infections $\left(\chi^{2}=8.6 ; P<0.005 ;\right.$ OR $\left.=8 \cdot 6\right)$ (Fig. 2).

Among $\mathrm{HPeV}$ mono infected children, 27(35.5\%) of them were females and 50(64.9\%) were males. The female to male ratio for $\mathrm{HPeV}$ infection was $1: 1.85$ (Table 1). The HPeV incidence was documented throughout the year with a low prevalence $(<10 \%)$ observed during the spring season (March and April).

\section{Clinical features of $\mathrm{HPeV}$ infection among children with acute gastroenteritis}

Among the $\mathrm{HPeV}$ infected children, 37.7\%, 44.2\% and $18.2 \%$ of the children showed clinical features such as no fever, low-grade fever and high-grade fever, respectively according to the vesikari clinical severity scoring method [20]. As compared with the children infected with other enteric viruses $\left(\chi^{2}=6.8 ; P<0.05 ; \mathrm{OR}=\right.$ $2 \cdot 6)$ and children without any viral infections investigated in this study $\left(\chi^{2}=5 \cdot 2 ; P<0 \cdot 05 ; \mathrm{OR}=2 \cdot 2\right)$, significantly more number of children with high- grade fever was recorded in $\mathrm{HPeV}$ infection (Table 1). Among the $\mathrm{HPeV}$ infected children, diarrhoea lasted for $1-12$ days (Mean $=3.0$ days) and the frequency of stool passage ranged from 3 to 25 times (Mean $=6 \cdot 0$ episodes) per day. Among the children infected with $\mathrm{HPeV}$ and other enteric viruses, diarrhoea lasted for 1-8 days (Mean $=3 \cdot 1$ days) and the frequency of stool passage ranged from 3 to 15 times $($ Mean $=5.7$ episodes) per day. Also, no significant difference was observed for different diarrheal condition between children infected with $\mathrm{HPeV}$, other enteric viruses and children without virus infection (Table 1).

In the study, 48 (62.3\%) children having HPeV mono infections and $40(67 \cdot 8 \%)$ children co-infected with $\mathrm{HPeV}$ and other enteric viruses experienced with vomiting. It was also observed that the vomiting lasted for 1-8 days (Mean $=2 \cdot 2$ days) and the frequency of vomiting episode ranged 2-8 (Mean $=3.4$ episode) per day. The frequency of 1-4 episodes of vomiting in a day was significantly higher in $\mathrm{HPeV}$ infected children $\left(\chi^{2}=3.8 ; \quad P<0.05 ; \quad\right.$ OR $\left.=1.6\right) \quad$ and other enteric virus-infected children $\left(\chi^{2}=6.9 ; P<0.05 ; \mathrm{OR}=1.4\right)$ as compared with the children tested negative for virus infection (Table 1). The disease condition based on Vesikari score was analysed and a significantly higher number of severe acute gastroenteritis cases was recorded in only $\mathrm{HPeV}$ infected children $(n=49$; $\left.\chi^{2}=4.6 ; P<0.05 ; \mathrm{OR}=1.8\right)$ and among the children with co-infection of $\mathrm{HPeV}$ and other enteric viruses $\left(n=40 ; \chi^{2}=6 \cdot 2 ; \quad P<0 \cdot 05 ; \quad\right.$ OR $\left.=2 \cdot 1\right)$ as compared with the children without virus infection (Table 1).

\section{Genotyping and identification of $\mathrm{HPeV}$ strains in acute gastroenteritis}

Out of the 136 specimens positive for HPeV-RNA, $102(75 \%)$ were genotyped. Of these, 72 strains were 
Table 1. Clinical and demographic features of human parechovirus (HPeV) infections among children with acute gastroenteritis

\begin{tabular}{|c|c|c|c|c|c|c|c|c|}
\hline Characteristic features & $\begin{array}{l}\text { Ot_Ent } \\
(n=372)\end{array}$ & $\%$ & $\begin{array}{l}\mathrm{HPeV} \\
(n=77)\end{array}$ & $\%$ & $\begin{array}{l}\text { Co-Inf } \\
(n=59)\end{array}$ & $\%$ & $\begin{array}{l}\text { Negative } \\
(n=451)\end{array}$ & $\%$ \\
\hline Age in months (median) & $0 \cdot 5-60(12)$ & & $1-54(10)$ & & $2-48(12)$ & & $0 \cdot 5-58 \cdot 5(12)$ & \\
\hline Male:Female & $1 \cdot 78: 1$ & & $1 \cdot 85: 1$ & & $2 \cdot 1: 1$ & & $1.69: 1$ & \\
\hline \multicolumn{9}{|l|}{ Fever } \\
\hline No fever & 158 & $42 \cdot 5$ & 29 & $37 \cdot 7$ & 27 & $45 \cdot 8$ & 194 & $41 \cdot 4$ \\
\hline Low $\left(37 \cdot 5-38 \cdot 5^{\circ} \mathrm{C}\right)$ & 185 & $49 \cdot 7$ & 34 & $44 \cdot 2$ & 25 & $42 \cdot 4$ & 217 & $49 \cdot 4$ \\
\hline $\operatorname{High}\left(>38 \cdot 5^{\circ} \mathrm{C}\right)$ & 29 & $7 \cdot 8$ & 14 & $18 \cdot 2$ & 7 & $11 \cdot 9$ & 40 & $9 \cdot 2$ \\
\hline \multicolumn{9}{|l|}{ Diarrhoea } \\
\hline 3-5 episode/day & 172 & $46 \cdot 2$ & 38 & $49 \cdot 4$ & 31 & $52 \cdot 5$ & 237 & $52 \cdot 5$ \\
\hline$\geqslant 6$ episode/day & 200 & $53 \cdot 8$ & 39 & $50 \cdot 6$ & 28 & $47 \cdot 5$ & 214 & $47 \cdot 5$ \\
\hline \multicolumn{9}{|l|}{ Duration } \\
\hline 1-5 days & 362 & $97 \cdot 3$ & 73 & $94 \cdot 4$ & 57 & $96 \cdot 6$ & 438 & $97 \cdot 1$ \\
\hline$\geqslant 6$ days & 10 & $2 \cdot 7$ & 4 & $5 \cdot 2$ & 2 & $3 \cdot 4$ & 13 & $2 \cdot 9$ \\
\hline \multicolumn{9}{|l|}{ Vomiting } \\
\hline No vomiting & 124 & $33 \cdot 3$ & 29 & $37 \cdot 7$ & 19 & $32 \cdot 2$ & 200 & $44 \cdot 1$ \\
\hline 1-4 episode/day & 186 & $50 \cdot 0$ & 41 & $53 \cdot 2$ & 27 & $45 \cdot 8$ & 183 & $40 \cdot 5$ \\
\hline$\geqslant 5$ episodes/day & 62 & $16 \cdot 7$ & 7 & $9 \cdot 1$ & 13 & $22 \cdot 0$ & 68 & $15 \cdot 4$ \\
\hline \multicolumn{9}{|l|}{ Duration } \\
\hline 1-2 days & 181 & $48 \cdot 6$ & 38 & $49 \cdot 4$ & 28 & $47 \cdot 5$ & 176 & $39 \cdot 8$ \\
\hline$\geqslant 3$ days & 67 & $18 \cdot 0$ & 10 & $13 \cdot 0$ & 12 & $20 \cdot 3$ & 75 & $17 \cdot 0$ \\
\hline
\end{tabular}

Ot Ent, other enteric viruses; Co-Inf, co-infection.

successfully amplified and sequenced for full VP1 (880 bp) gene and the remaining 30 strains for partial VP1 (658 bp) gene. Phylogenetic analysis identified a total of 13 genotypes among the 102 strains, based on the criterion described earlier (22). The most prevalent genotypes observed were of HPeV1 (39, 38.2\%), HPeV3 (13, 12.7\%), HPeV4 (13, 12.7\%) and HPeV5 $(11,10,7 \%)$. Other nine HPeV types which included HPeV14 (4), HPeV2 (4), HPeV8 (4), HPeV10 (3), HPeV6 (3), HPeV13 (3), HPeV7 (2), HPeV16 (2) and HPeV11 (1) also were detected at lower level.

The strains identified as $\mathrm{HPeV} 1$ were grouped into two clusters i.e., HPeV1A $(84.6 \%, 33 / 39)$ and HPeV1B $(15 \cdot 4 \%, 6 / 39)$. The nucleotide (NT) identity between the HPeV1A Indian strains and the prototype strain (Harris strain, 1956) was noted as 80.58-84.02\%. The six strains in HPeV1B showed $90 \cdot 35-92 \cdot 37 \%$ NT identity with the strains from the Netherlands, AB112487 and FM178558 (Fig. 3).

Among the Indian strains HPeV1A and HPeV1B, NT identities were found to be in the range $74 \cdot 09-78 \cdot 76 \%$. All the $13 \mathrm{HPeV} 3$ strains formed in two separate clusters of which a cluster of five strains showed $91 \cdot 83-94 \cdot 36 \%$ NT homology with the strains from the Netherlands (GQ183029). Remaining eight strains formed into a separate cluster and showed $77 \cdot 31-79 \cdot 31 \%$ nucleotide identity with the HPeV3 strains from Japan (AB759191) and the Netherlands (GQ183029). NT homology between these two Indian clusters ranged into $76 \cdot 3-78 \cdot 6 \%$. Of the $13 \mathrm{HPeV} 4$ strains, 11 of them clustered closely with Bolivian strains and showed $79 \cdot 84-81 \cdot 97 \%$ NT identity; other two strains showed highest (93.08-94.43\%) NT identity with the strains from France, Sri Lanka and the Netherlands. Two separate clusters of HPeV4 study strains showed 76.46$81 \cdot 63 \%$ NT identity between them. Also, HPeV5 detected in 11 samples, among these 6 strains clustered with the strains isolated in 2004 from the Netherlands (FJ373175) and showed 81.51-83.29\% NT identity and remaining cluster of five strains showed $78 \cdot 88-82 \cdot 02 \%$ NT identity with the Chinese and the Netherlands (EU077511) strains. Two different clusters showed $78 \cdot 81-82 \cdot 67 \%$ NT identity between them.

The results of deduced amino acid sequence alignment indicated that all the Indian strains contained the highly conserved $\mathrm{Q} / \mathrm{N}$ and $\mathrm{Q}$ or $\mathrm{E} / \mathrm{S}$ which are believed to be the putative cleavage site of VP3/VP1 and VP1/2A, respectively. All the HPeV1 and 2 strains identified in the present study showed the presence of Arginylglycylaspartic acid (RGD) motif. Interestingly, RGD motif was not found in 7/13 strains of HPeV4 and $2 / 2$ strains of HPeV6 detected. 


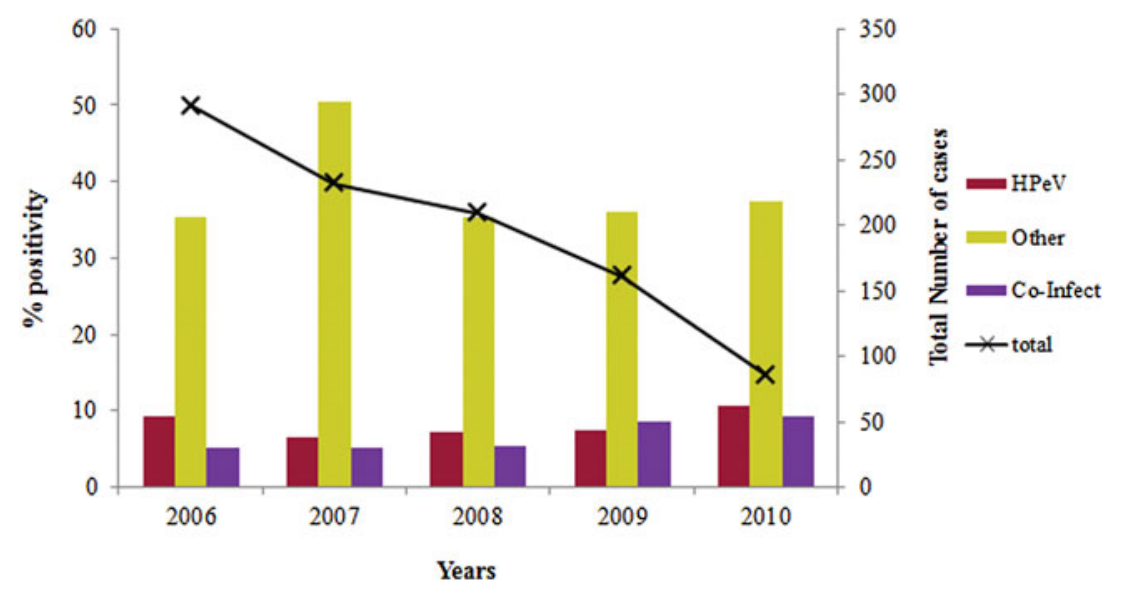

Fig. 1. Year wise distribution of $\mathrm{HPeV}$ in acute gastroenteritis cases during 2006-2010.

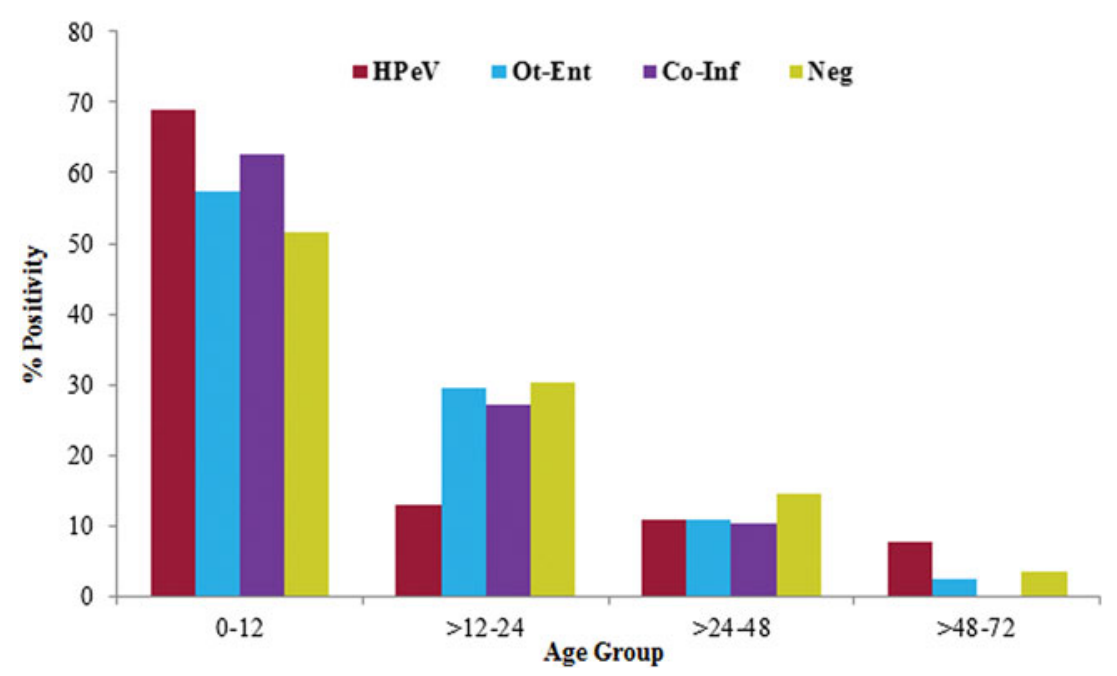

Fig. 2. The age distribution of $\mathrm{HPeV}$ positivity in acute gastroenteritis cases.

\section{Clinical features of $\mathrm{HPeV}$ genotypes in acute gastroenteritis}

HPeV1 was detected predominantly and significantly showed severe infections $(30 \%)$ as compared with HPeV3, 4 and 5 in acute gastroenteritis cases. HPeV6, 13, 14 and 16 types were found to have severe infections only albeit low frequency of detection. HPeV5 and 11 showed mild to moderate infections. HPeV1 and 3 showed the highest rate of single infection $(36 \%$ and $11.69 \%)$ as compared with $\mathrm{HPeV} 4$ and 5 which have been detected predominantly in mixed infections (10.2\% and 8.5\%). HPeV2 which was detected rarely, $\mathrm{HPeV} 7,11$ and 16 types attributed to mono infections only in comparison with HPeV13 which was found to show mixed infection. Majority of the HPeV1 and 5 infections were detected in children $\leqslant 1$ year age group in the acute gastroenteritis cases (Fig. 4).

\section{DISCUSSION}

HPeVs show a worldwide distribution, however, there have been no strain surveillance studies available on HPeVs in acute gastroenteritis in India. The data generated in this study showed $13.9 \%$ of $\mathrm{HPeV}$ prevalence in patients with acute gastroenteritis. This is further supported by the studies from other Asian countries reporting $8 \cdot 1-29 \cdot 4 \% \mathrm{HPeV}$ positivity in sporadic cases of acute gastroenteritis [19, 25]. Most of the $\mathrm{HPeV}$ infections were detected among the children of $<2$ years age and the number was highest in the 1 st year of life. This is in agreement with the 


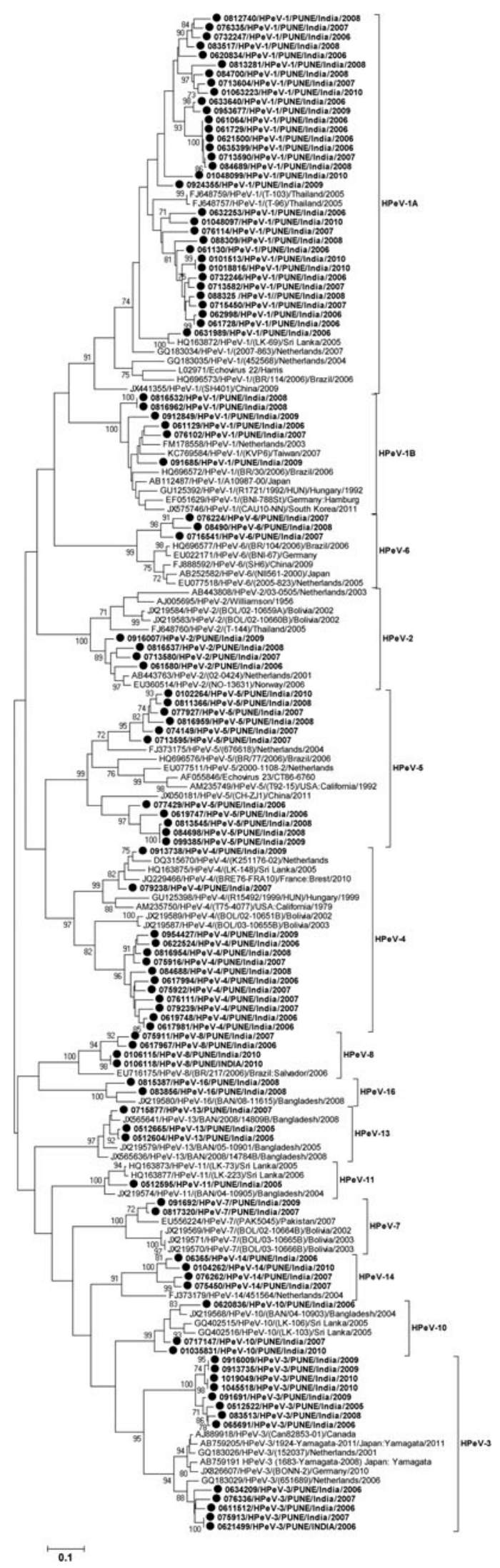

Fig. 3. Phylogenetic dendrogram on the basis of VP1 gene of $\mathrm{HPeV}$ strains. Study strains are highlighted in bold and indicated with circle (O). The scale indicates genetic distance. previous studies reported that majority of the $\mathrm{HPeV}$ infections were observed below 2 years of age $[4,26]$. Majority of the $\mathrm{HPeV}$ infections noted in the study were found in male patients. The studies conducted previously indicated a need for further investigation to understand the difference if any, in susceptibility between the two genders [27].

Co-infections with $\mathrm{HPeV}$ and other enteric viruses $(43.4 \%)$ noted in this study have also been reported from China and Sri Lanka $(64 \cdot 4-66 \cdot 7 \%)[28,29]$ suggesting that dual infection is a possible mode of infection. The year wise prevalence of $\mathrm{HPeV}$ was reported to be $9.6 \%$ and $10.7 \%$ in acute gastroenteritis cases during 2006 and 2010 respectively.

In the present study, a total of 13 different $\mathrm{HPeV}$ genotypes were detected among Indian children with acute gastroenteritis which suggests circulation of heterogeneous $\mathrm{HPeV}$ genotypes. Month-wise distribution of HPeVs in acute gastroenteritis cases could not be analysed as wide range of $\mathrm{HPeV}$ types were under circulation and not detected in each month of the year. Therefore, the study has been restricted only to the year wise distribution of HPeVs in acute gastroenteritis cases. Studies reported from other countries have shown that HPeV1 as the predominant genotype in children with acute gastroenteritis $[25,28,30]$. Phylogenetic analysis of the $\mathrm{HPeV} 1$ sequences demonstrating the existence of two clusters, cluster 1B of the contemporary strains and cluster 1 A related to the prototype Harris strain was in agreement with the results of the previous study [4]. The circulation of HPeV1A strains as revealed by our study in India differed from the other Asian countries reporting mainly infections with HPeV1B strains [19, 25, 28].

In the present study, $\mathrm{HPeV} 3$ is the second most predominant genotype detected. The biennial cycle, a distinctive characteristic of $\mathrm{HPeV} 3$ has been reported previously [21, 28]. However, in this study HPeV3 was detected throughout the year. Also, HPeV3 detected previously in younger age group [31] was found in children of a higher age in the present study which is in agreement with the study reported earlier from China [19]. Based on the data on HPeV3 occurrence indicates that HPeV3 might have different epidemiological features associated with different geographical regions. In the phylogenetic analysis of full length VP1 gene, eight $\mathrm{HPeV} 3$ strains formed in to a separate cluster and also showed $76 \cdot 3-78 \cdot 6 \%$ NT and $84-86 \cdot 8 \%$ AA identity with other HPeV3 strains which is less than the cutoff value described previously for genotyping of HPeVs [22], could suggest the circulation of HPeV3 variant in Pune, western India. 


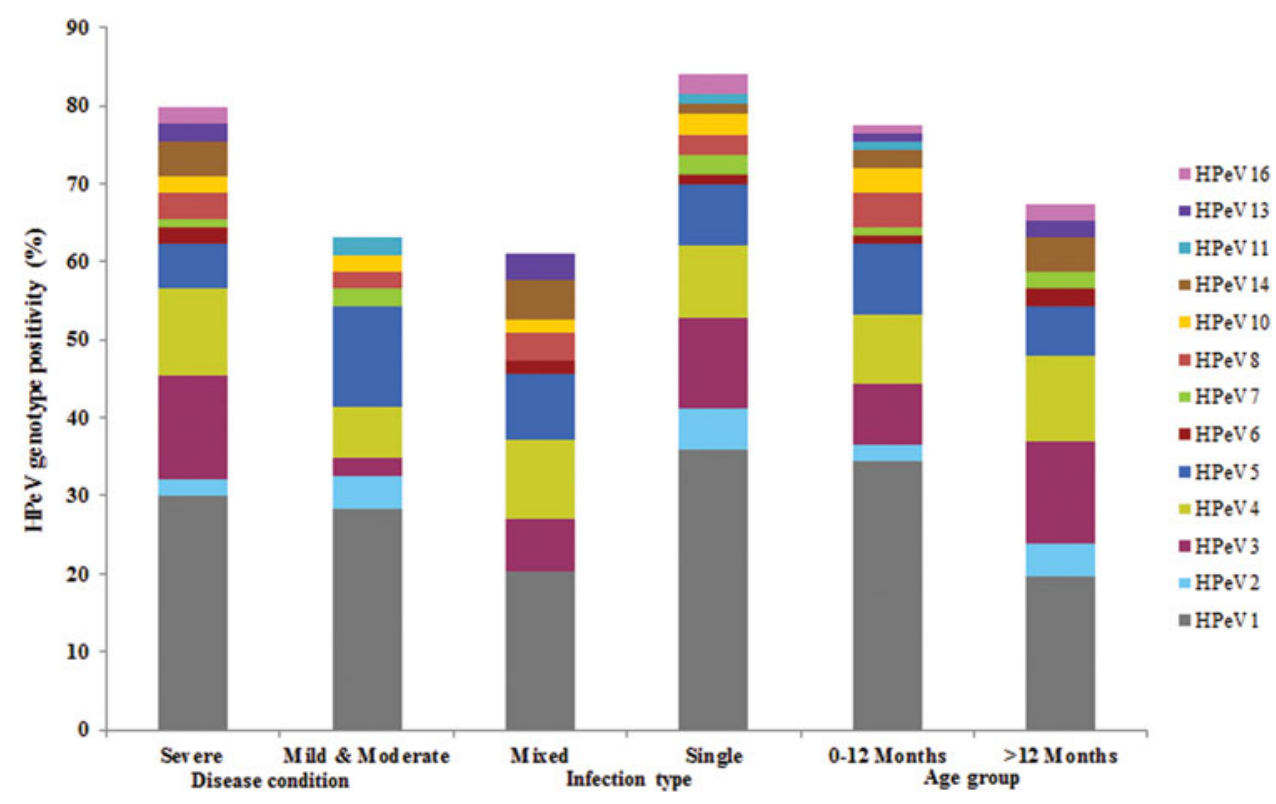

Fig. 4. HPeV Genotype positivity and its correlation with clinical features in acute gastroenteritis patients.

Interestingly, $\mathrm{HPeV} 2$ reported as a rare genotype and mostly in association with gastrointestinal symptoms was identified during 2006-2009 but not in 2010 [32].

Also, failure to genotype $25 \%$ of the HPeV strains could be due to mismatching of the primer sets utilised in the study or low titers of virus in the clinical samples.

A Q/N conservation in the VP3/VP1 cleavage site was noted in all the sequences of $\mathrm{HPeV}$ strains identified in the study, which corroborated with the report from the previous study and the preference for Glutamine (Q) at the $\mathrm{P} 1$ position [9]. The $\mathrm{C}$ terminal region of VP1 gene contains an RGD motif found essential for replication of HPeV1,2,4,5 and 6 and attachment to cell surface fibronectins $\alpha \mathrm{v} \beta 1$ and $\alpha v \beta 3$ however, other types have been found to lack this surface determinant [33]. Lack of RGD motif supports the presence of an alternative pathway of cell entry and infection [34]. Also, HPeV4 strains identified in this study showed the circulation of two variants in Indian population one utilising RGD motif and other an alternative RGD independent pathway for cell entry. The occurrence of such variants that belong to the same genotype is common in the family picornaviridae.

Although the role of $\mathrm{HPeV}$ in causing acute gastroenteritis has been studied by several investigators, no conclusive data are available to date $[19,35]$. In the present study, HPeVs were detected frequently amongst acute gastroenteritis cases. These data need to be ascertained in acute gastroenteritis patients from wider geographic regions in India for a better understanding of epidemiology and genetic diversity of HPeVs. Further, case-control studies conducted to find out the presence and load of these viruses in the faeces will help to determine their association with diarrhoea.

In summary, the current study identified $\mathrm{HPeV}$ genotypes that are circulating in Asian or other countries to date. Also RGD absent' HPeV5 and 6 strains and co-circulation of two variant strains of HPeV4 in Indian population are reported. The present study highlights the detection and characterisation of a wide spectrum of $\mathrm{HPeV}$ genotypes in acute gastroenteritis patients for the first time from India.

\section{ACKNOWLEDGEMENTS}

We are grateful to Dr D.T. Mourya, Director, National Institute of Virology, Pune (India) for his constant support. We also acknowledge the cooperation extended by Drs. R. Dhongade, V. Kalrao and A.R. Bavdekar for providing clinical specimens. No external financial support was used in conducting this study.

\section{DECLARATION OF INTEREST}

None. 


\section{REFERENCES}

1. Wigand R, Sabin AB. Properties of ECHO types 22, 23 and 24 viruses. Arch Gesamte Virusforsch 1961; 11: 224-227.

2. Stanway G, Hyypia T. Parechoviruses. Journal of Virology 1999; 73: 5249-5254.

3. Stanway G, Joki-Korpela P, Hyypia T. Human parechoviruses-biological and clinical significance. Reviews in Medical Virology 2000; 10: 57-69.

4. Baumgarte $\mathbf{S}$, et al. Prevalence, types and RNA concentrations of human parechoviruses, including a sixth parechovirus type, in stool samples from patients with acute enteritis. Journal of Clinical Microbiology 2008; 46: $242-248$.

5. Benschop KS, et al. Fourth human parechovirus serotype. Emerging Infectious Diseases 2006; 12: 1572-1575.

6. Ehrnst A, Eriksson M. Echovirus type 23 observed as a nosocomial infection in infants. Scandinavian Journal of Infectious Diseases 1996; 28: 205-206.

7. Ito $\mathbf{M}$, et al. Isolation and identification of a novel human parechovirus. Journal of General Virology 2004; 85: 391-398.

8. Oberste MS, Maher K, Pallansch MA. Complete sequence of echovirus 23 and its relationship to echovirus 22 and other human enteroviruses. Virus Research 1998; 56: 217-223.

9. Watanabe $\mathbf{K}$, et al. Isolation and characterization of novel human parechovirus from clinical samples. Emerging Infectious Diseases 2007; 13: 889-895.

10. Li L, et al. Genomic characterization of novel human prechovirus type. Emerging Infectious Diseases 2009; 15: $288-291$.

11. Drexler JF, et al. Novel human parechovirus from Brazil. Emerging Infectious Diseases 2009; 15: 310-313.

12. Nix WA, et al. Diversity of picornaviruses in rural Bolivia. Journal of General Virology 2013; 94: 20172028.

13. Elliott EJ. Acute gastroenteritis in children. British Medical Journal 2007; 334: 35-40.

14. Kang G, et al. Diversity of circulating rotavirus strains in children hospitalized with diarrhea in India, 20052009. Vaccine 2013; 31: 2879-2883.

15. Chhabra $\mathbf{P}$, et al. Epidemiological, clinical and molecular features of norovirus infections in western India. Journal of Medical Virology 2009; 81: 922-932.

16. Verma H, Chitambar SD, Gopalkrishna V. Identification and characterization of enteric adenoviruses in infants and children hospitalized for acute gastroenteritis. Journal of Medical Virology 2009; 81: 60-64.

17. Verma H, Chitambar SD, Gopalkrishna V. Astrovirus associated acute gastroenteritis in western India: predominance of dual serotype strains. Infection, Genetics and Evolution 2010; 10: 575-579.

18. Verma H, Chitambar SD, Gopalkrishna V. Circulation of Aichi virus genotype B strains in children with acute gastroenteritis in India. Epidemiology and Infection 2011; 139: 1687-1691.
19. Zhang DL, et al. Prevalence of human parechovirus in Chinese children hospitalized for acute gastroenteritis. Clinical Microbiology and Infection 2011; 17: 1563-1569.

20. Ruuska T, Vesikari T. Rotavirus disease in Finnish Children: use of numerical scores for clinical severity of diarrhoeal episodes. Scandinavian Journal of Infectious Diseases 1990; 22: 259-267.

21. van der Sanden S, et al. Prevalence of human parechovirus in the Netherlands in 2000 to 2007. Journal of Clinical Microbiology 2008; 46: 2884-2889.

22. Nix WA, et al. Parechovirus typing in clinical specimens by nested or semi-nested PCR coupled with sequencing. Journal of Clinical Virology 2010; 48: 202-207.

23. Sapkal GN, et al. Enteroviruses in patients with acute encephalitis, Uttar Pradesh, India. Emerging Infectious Diseases 2009; 15: 295-298.

24. Tamura K, et al. MEGA5: molecular evolutionary genetics analysis using maximum likelihood, evolutionary distance, and maximum parsimony methods. Molecular Biology and Evolution 2011; 28: 2731-2739.

25. Pham NTK, et al. Detection of human parechovirus in stool samples collected from children with acute gastroenteritis in Japan during 2007-2008. Journal of Medical Virology 2011; 83: 331-336.

26. Abed Y, Boivin G. Human parechovirus types 1,2 and 3 infections in Canada. Emerging Infectious Diseases 2006; 12: 969-975.

27. Ito M, et al. Detection of human parechoviruses from clinical stool samples in Aichi, Japan. Journal of Clinical Microbiology 2010; 48: 2683-2688.

28. Guo Y, Duan Z, Qian Y. Changes in human parechovirus profiles in hospitalized children with acute gastroenteritis after a three year interval in Lanzhou, China. PLoS ONE 2013; 8: e68321.

29. Pham NT, et al. Human parechovirus infections in children hospitalized with acute gastroenteritis in Sri Lanka. Journal of Clinical Microbiology 2011; 49: 364-366.

30. Han TH, et al. Detection of human parechoviruses in children with gastroenteritis in South Korea. Archives of Virology 2011; 156: 1471-1475.

31. Benschop KS, et al. High prevalence of human parechovirus genotypes in the Amsterdam region and identification of specific $\mathrm{HPeV}$ variants by direct genotyping of stool samples. Journal of Clinical Microbiology 2008; 46: 3965-3970.

32. Pham NTK, et al. Diversity of human parechoviruses isolated from stool samples collected from Thai children with acute gastroenteritis. Journal of Clinical Microbiology 2010; 48: 115-119.

33. Wildenbeest JG, et al. The need for treatment against human parechoviruses: how, why and when? Expert Review of Anti-Infective Therapy 2010; 8: 1417-1429.

34. Alam MM, et al. Identification of human parechovirus genotype, $\mathrm{HPeV}-12$, in a paralytic child with diarrhea. Journal of Clinical Virology 2012; 55: 339-342.

35. Tapia G, et al. Longitudinal observation of parechovirus in stool samples from Norwegian infants. Journal of Medical Virology 2008; 80: 1835-1842. 